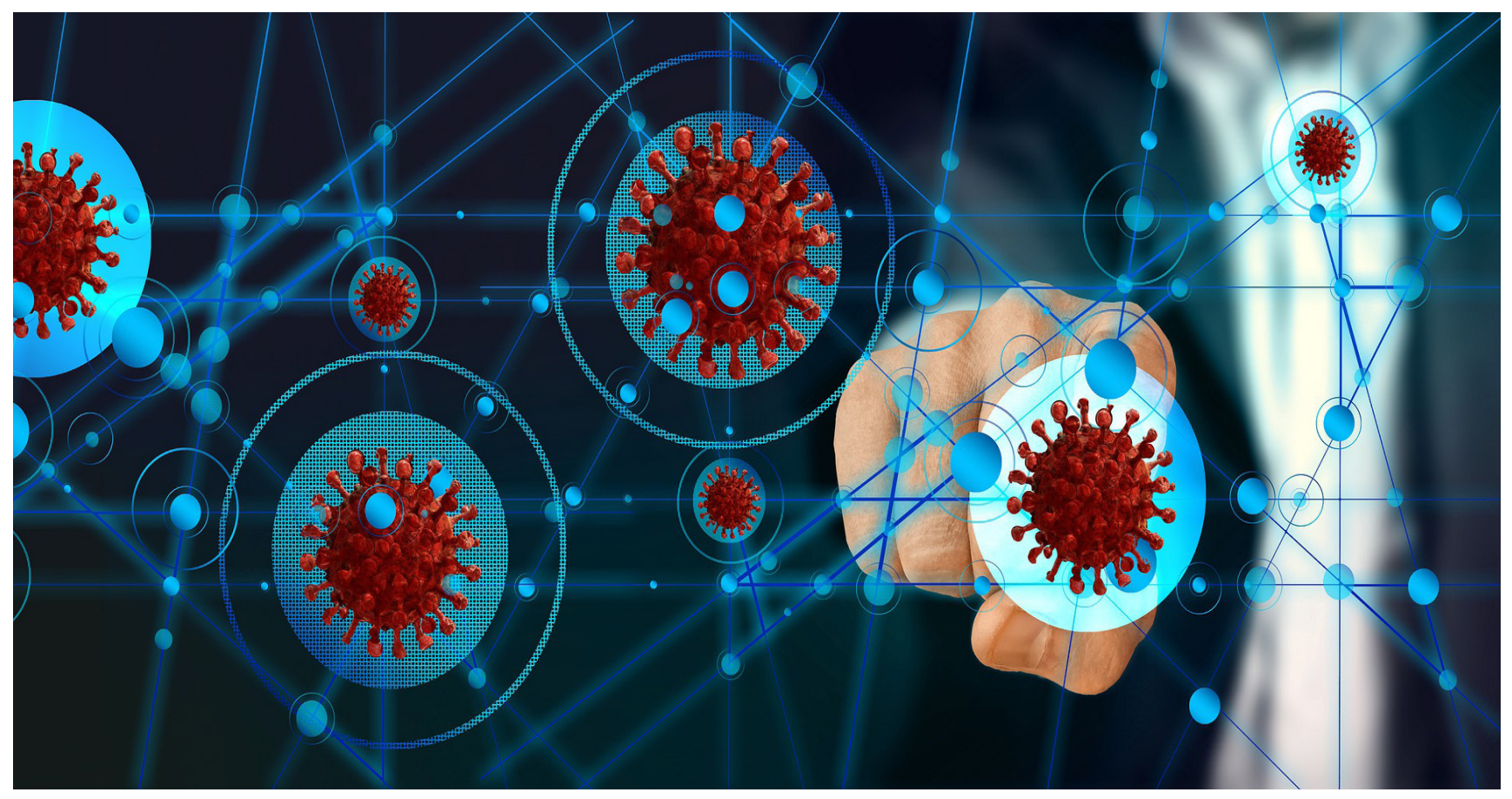

\title{
Impacto del covid-19 en el sector turismo en Colombia
}

\section{Impact Of Covid-19 On The Tourism Sector In Colombia}

\section{Ronald Edwin Contreras}

Estudiantes Administración Turística y Hotelera, Fundación de Estudios Superiores Comfanorte FESC, Cúcuta, Colombia

Lisset Katherine Serrano-Villamizar

Estudiantes Administración Turística y Hotelera, Fundación de

Estudios Superiores Comfanorte FESC, Cúcuta, Colombia

\section{Enmanuel Omar Nava-Sarmiento}

Docente, aduanera@fesc.edu.co, Fundación de Estudios Superiores Comfanorte FESC, Cúcuta, Colombia. 
Cómo citar: Contreras, R.E., Serrano-Villamizar, L.K., Nava-Sarmiento, E.O. (2020). Impacto del covid-19 en el sector turismo en Colombia. Reflexiones contables (Cúcuta), 3 (2), 77-84

\section{Resumen}

La epidemia mundial de coronavirus ocasionará efectos importantes en la economía global durante los primeros trimestres de 2020, lo cual ha hecho que impacte negativamente la economía global, el COVID-19 no es solo una emergencia para la salud pública, sino que también implica un choque económico tanto de oferta como de demanda, por ello se ve la necesidad de determinar el impacto del COVID-19 en el sector turismo en Colombia. La investigación se desarrolló por medio de una revisión bibliográfica en la cual, críticamente, se analizaron los datos relacionados con el fenómeno de interés después de realizar una búsqueda en las principales bases de datos. En Colombia si la pandemia se controla en uno o dos meses se prevé que la economía colombiana crecerá un $2,3 \%$, en un término a medio plazo (4 meses) se prevé que la economía crecerá 1,2\% sin embargo en el peor de los panoramas si se resuelve a un plazo mayor a 6 meses se prevé que el crecimiento será un $0,4 \%$ y a la baja; el gobierno nacional ha tomado medidas mediante decretos para poder proteger sus sectores financieros y que el PIB no se tan devastador para la economía del país entre ellas están los decretos 462, 397, 557 y 417; las alianzas entre secretarias departamentales y municipales podrían generar propuestas innovadores para que la crisis en el sector turismo se reactive de manera positiva para todos. Esta pandemia ha dejado a casi todas las ramas de actividad de la nación perjudicadas, especialmente al sector turismo, en donde se ha visto una disminución contundente en el PIB de la nación incluso algunos negativos.

Palabras clave: COVID-19, Crisis económica, Sector turismo

\section{Abstract}

Introduction: the global coronavirus epidemic will cause significant effects on the global economy during the first quarters of 2020, which has made it negatively impact the global economy, COVID-19 is not only an emergency for public health, but also implies An economic shock, both in supply and demand, therefore the need to determine the impact of COVID-19 on the tourism sector in Colombia is seen. Materials and methods: The research was developed through a bibliographic review in which, critically, the data related to the phenomenon of interest was analyzed after performing a search in the main databases. Results: in Colombia, if the pandemic is controlled in one or two months, it is expected that the Colombian economy will grow $2.3 \%$, in the medium term (4 months) the economy is expected to grow $1.2 \%$, however in the worst of the scenarios if it is resolved over a period of more than 6 months, growth is expected to be $0.4 \%$ and downward; the national government has taken measures through decrees in order to protect its financial sectors and that the GDP is not so devastating for the country's economy, among them are decrees 462, 397, 557 and 417; alliances between departmental and municipal secretaries could generate innovative proposals so that the crisis in the tourism sector is reactivated in a positive way for all. Conclusion: This pandemic has left almost all branches of activity in the nation affected, especially the tourism sector, where a sharp decrease in the nation's GDP has been seen, even some negatives.

Key words: COVID-19, Economic crisis, Tourism sector 


\section{Introducción}

La reciente crisis por la epidemia mundial de coronavirus ocasionará efectos importantes en la economía global durante los primeros trimestres de 2020. Aunque todavía es muy pronto para evaluar los alcances de la enfermedad y sus efectos en la economía, se muestran algunas de las posibles implicaciones y medidas políticas que diversos especialistas e instituciones financieras han vertido sobre el tema. La pandemia de COVID-19 está afectando negativamente a la economía global y parece que sus efectos se mantendrán al menos durante los dos primeros trimestres de 2020. Representa una emergencia para la salud pública e implica un choque económico tanto de oferta como de demanda; es decir, que está afectando tanto a productores como a consumidores. A nivel mundial, la pandemia del coronavirus COVID-19 podría ocasionar que la economía mundial se reduzca un 0,9\% durante el 2020, en vez de crecer hasta el 2,5\% como se preveía, destaca una nota informativa del Departamento de Asuntos Económicos y Sociales de la ONU (López, 2020).

La comisión económica para América latina y el caribe estima una contracción de $-1,8 \%$ del producto interno bruto regional, lo que podría llevar a que el desempleo en la región suba en diez puntos porcentuales. Esto llevaría a que, de un total de 620 millones de habitantes (América latina y el caribe), el número de pobres en la región suba de 185 a 220 millones de personas; en tanto que las personas en pobreza extrema podrían aumentar de 67,4 a 90 millones. (CEPAL,2020).

La crisis que está generando el COVID-19 afecta a todos los sectores especialmente el turismo, pues ha ocasionado que muchos de los planes turísticas que ya se encontraban planeados para la población en general fueran cancelados o aplazados para fechas indeterminadas ocasionando que este sector sea el más perjudicado, teniendo en cuenta que no ha recibido ningún tipo de ingreso por más de tres meses consecutivos, haciendo que pequeñas y medianas empresas se vean en la obligación de suspender contratos indefinidamente hasta nueva orden. Esta pandemia ha puesto en jaque a grandes cantidades de empresarios que están tratando de salir a flote, pues no solo son las agencias de viajes, es un conjunto de hoteles, restaurantes, guías y demás trabajadores que directa o indirectamente se benefician de este sector, y que lo ven como un medio de trabajo sustentable hasta este momento, provocando que el país se encuentra aún más sumergido en la pobreza y el PIB caiga de la misma manera (Pulido Arbelaez \&Cabello Beltrán, 2020)

Por ello se hace necesario determinar cuál será el impacto que el COVID-19 tendrá para el sector turismo en el país, describir las medidas que el gobierno nacional ha tomado para contrarrestar la crisis y que propuestas innovadoras hechas por expertos se pueden implementar como medida de contingencia en la crisis.

\section{Materiales y métodos}

La investigación se desarrolló por medio de una revisión bibliográfica en la cual, críticamente, se analizaron los datos relacionados con el fenómeno de interés después de realizar una búsqueda en las principales bases de datos nacionales e internaciones, mejorándola a través de palabras claves como COVID-19, crisis económica, sector turismo. 


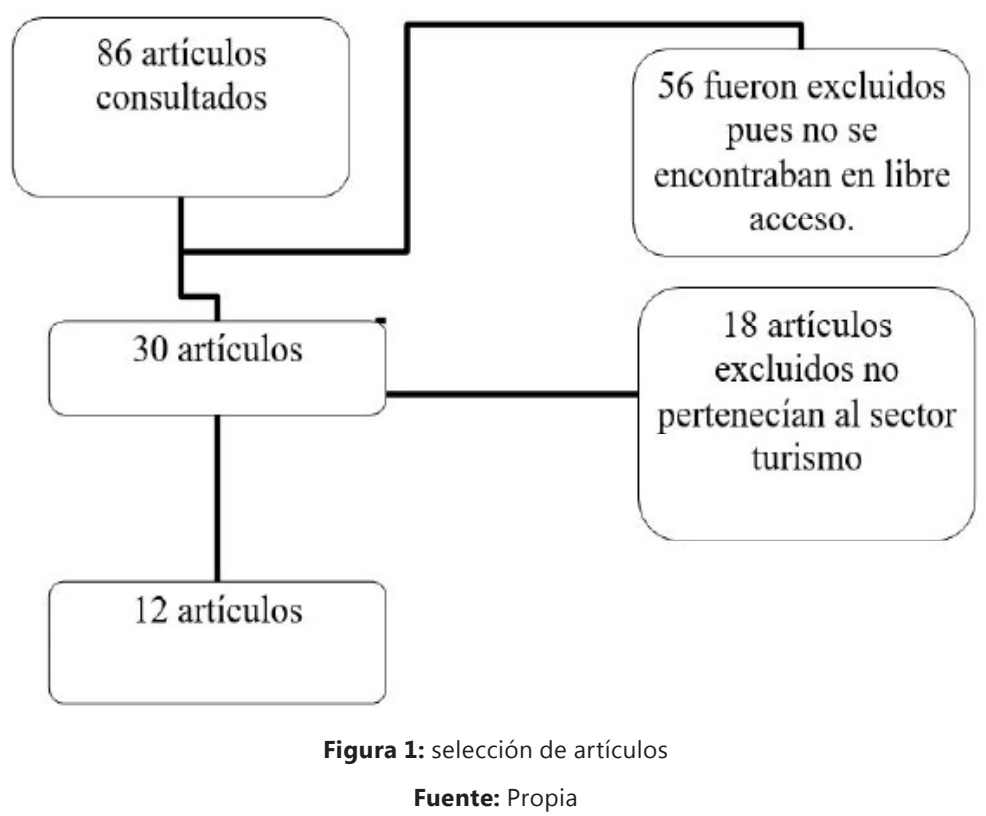

\section{Resultados y análisis}

\section{El impacto del COVID-19 a nivel nacional.}

En Colombia se esperaba para este 2020 la economía aumentará 3,2\%, debido a que todo indicaba que la economía sería similar a la del 2019, aunque el panorama ahora es totalmente contradictorio, en el mejor de los casos si la pandemia se controla en uno o dos meses se prevé que la economía colombiana crecerá un $2,3 \%$, en un término a medio plazo (4 meses) se prevé que la economía crecerá $1,2 \%$ sin embargo en el peor de los panoramas si se resuelve a un plazo mayor a 6 meses se prevé que el crecimiento será un 0,4\% y a la baja. (Fedesarrollo, 2020).

El pronóstico del PIB de Colombia para el 2020 en porcentaje de acuerdo a la ANDI durante el escenario de la pandemia mundial se observa que todos los sectores se espera que se encuentren a la baja frente al 2019, pero habrá algunos sectores mucho más golpeados que otros, sectores como actividades financieras, actividades empresariales, actividades inmobiliarias, actividades de recreación, minería, industria y comercio, transporte y turismo se encuentran muy por debajo del escenario del escenario observado en 2019, sin embargo existe un sector el cual se prevé crecimiento y es el sector agropecuario. (Figura 2)

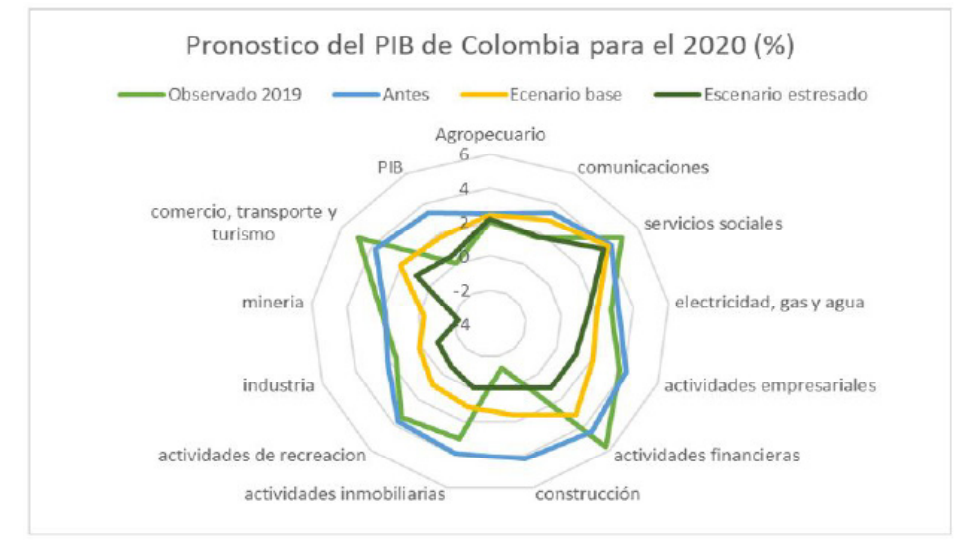

Figura 2. Pronostico del PIB de Colombia para el 2020 (\%)

Fuente: Propia, datos tomados de la ANDI 
El ingreso de los colombianos ha caído alrededor en 5,8 billones de pesos en marzo y abril por cuenta de las medidas para enfrentar el coronavirus, según cálculos de Anif. Esta entidad advirtió que dicha cifra podría aumentar hasta los 12,5 a 26,9 billones (1,1 a 2,4 como porcentaje del PIB) en caso de que el aislamiento permanezca hasta mayo o junio. En ese caso, cada mes representaría menos ingreso laboral por el orden de 14,5 billones adicionales, lo que conlleva a que menor ingresos por parte de los colombianos, menos demanda.

En el mes de marzo, las ventas a través plataformas electrónicas cayeron $13,8 \%$, según datos de la alianza Fenalco-CredibanCo, Mientras los pagos electrónicos para comprar alimentos en supermercados y grandes cadenas crecieron $79 \%$ las droguerías repuntaron $29 \%$, las compras de pasajes aéreos presentaron un desplome del $68 \%$. Las de vestuario cayeron $48 \%$ mientras que las de estaciones de servicio lo hicieron un $8,49 \%$. (Instituto Belisario Domínguez, 2020).

La Cámara Colombiana de Comercio Electrónico y el Ministerio de las TIC, junto con algunas empresas afiladas al gremio, han recopilado información para entender cómo se ha comportado este segmento de la economía digital en Colombia durante la pandemia. Respecto a la última semana de febrero, el valor total de las compras realizadas y pagadas a través de internet en Colombia cayó el $47 \%$ en la última semana de marzo, aunque presentó un crecimiento semanal del $29 \%$ en la primera semana de abril, una vez se dio claridad por parte del Gobierno Nacional en la operación de las plataformas de comercio electrónico en medio del aislamiento preventivo obligatorio. (CCCE, 2020).

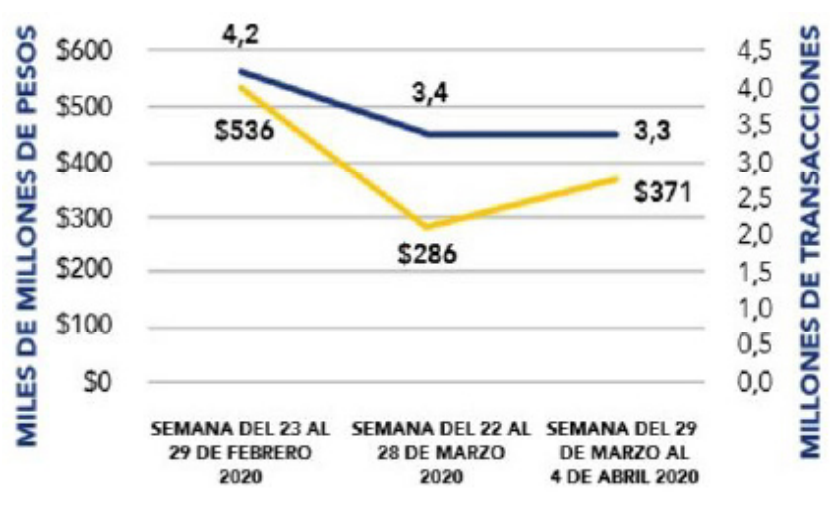

\footnotetext{
VENTAS TRANSACCIONES

Figura 3. Transacciones electrónicas en el comercio nacional

Fuente: Credibanco, Redeban y $\mathrm{ACH}$
}

El confinamiento obligatorio y la pérdida de empleos, especialmente en el sector informal, se traduce además en un choque de demanda, en donde los hogares reducen sus niveles de consumo. Este choque de demanda se agrava con la reducción en el ingreso disponible del país derivada de la caída en los precios internacionales del crudo, que profundiza la reducción del consumo público y privado. Esto último se vería reflejado en un menor dinamismo en sectores como el comercio, transporte, alojamiento y servicios de comida, actividades financieras, actividades de entretenimiento, y la industria manufacturera. En ese sentido, por el lado de la oferta el choque afectaría en mayor medida al sector de comercio y transporte $(-0,9 \%$ escenario medio vs $+4,5 \%$ escenario Prospectiva), la industria manufacturera $(-2,6 \%$ medio vs $2,4 \%$ en Prospectiva), este último especialmente en las actividades de refinación que se desacelerarían en 7,7\% frente al escenario base de Prospectiva $(-4,2 \%$ medio vs $+3,5 \%$ base); y el sector de la minería, que también vería reducido su generación de valor agregado al contraerse en $2,1 \%$ (vs $+2,3 \%$ en Prospectiva) obedeciendo al menor dinamismo en la actividad de extracción petrolera. (Fedesarrollo, 2020). (Figura 4) 


\section{2}

Siguiendo los escenarios simulados, las proyecciones correspondientes a la tasa de desempleo para cada caso. Estimamos que en el escenario medio la tasa de desempleo podría alcanzar 15,4\%, 4,9\% superior a lo observado en el promedio de 2019 (10,5\%). En el escenario optimista la tasa de desempleo cerraría en $13,3 \%$, mientras que en el escenario pesimista podría incluso alcanzar el 19,5\%. Estos ejercicios son indicativos y reflejan la importancia de hacer un seguimiento detallado a la tasa de desempleo durante los próximos meses (Fedesarrollo, 2020).

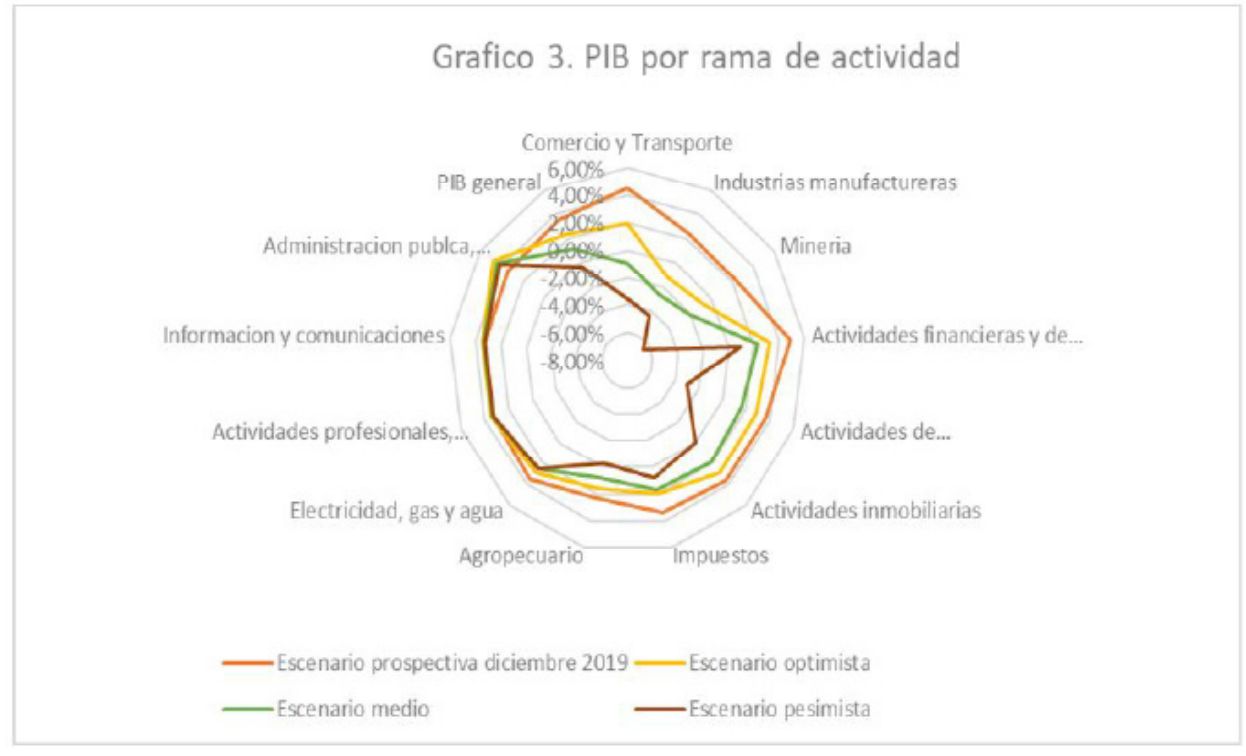

Figura 4. PIB por rama de actividad

Fuente: propia

Medidas tomadas por el gobierno Nacional para afrontar la emergencia económica.

El gobierno nacional ha tomado medidas mediante decretos para poder proteger sus sectores financieros y que el PIB no se tan devastador para la economía del país y de la misma manera esta pueda resurgir, dentro de estas medidas se encuentra la del decreto 462 del 22 de marzo del 2020 en donde se prohíbe la exportación y reexportación de productos necesarios para la confrontación de la pandemia, en donde el sector agropecuario solo producirá para abastecer a la nación, creando un situación autosostenible como factor principal para las necesidades primordiales de los colombianos. (MinCIT,2020).

Dentro del sector turismo el gobierno en su decreto 397 del 13 de marzo del 2020 establece un beneficio en la presentación y pago de la contribución parafiscal para la promoción del turismo hasta el 17 de junio del 2020, teniendo en cuenta que estos deben ser pagados por entidades en donde el sector económico sea netamente el turismo, sin embargo, esta situación no ha sido bien vista, puesto que solo es un plazo para la contribución del mismo más no una baja, ya que el sector turismo es uno de los más afectados, y que seguirá viéndose afectado al menos durante el 2020. (MinCIT,2020)

Sin embargo, en el decreto 557 del 15 de abril del 2020, se establece que los recursos de impuesto nacional con al turismo artículo 4 la Ley 1101 2006, se destinarán para contribuir a las empresas turísticas que cuenten con inscripción y vigente en el Registro Nacional Turismo y no para la promoción del mismo, lo que favorece directamente a Mundidestinos S.A. (MinCIT,2020) 
También, el gobierno nacional en el Decreto 417 del 17 de marzo de 2020 establece que se podrán incluir disposiciones que flexibilicen los plazos de pago de las obligaciones, pagos a los acreedores distintas clases de forma simultánea o sucesiva y mecanismos alivio financiero y reactivación empresarial, en donde se destinarán 14,8 billones de pesos para la reactivación empresarial con el fin de mitigar los efectos financieros en las medianas y pequeñas empresas debido a la emergencia sanitaria, respaldando los programas de garantías crediticias. (MinCIT,2020)

\section{Propuesta innovadora}

Una de las opciones del sector turismo es el proceso de innovación el cual podrá surgir según expertos en la materia a finales del 2020 y en su auge en 2021, en donde sin duda, el turismo interno será el primero en empezar su recuperación, por eso es importante la implementación de una campaña que incentive a los colombianos a recorrer nuevamente su país. Además, las ciudades turísticas de la nación impondrán medidas de seguridad y salubridad para que los colombianos puedan sentirse protegidos por las agencias de viajes en el entorno de la salud, retomando la confianza de los turistas.

Además, podría existir una alianza con las secretarias municipales, departamentales y nacionales de turismo, en donde ellos con alianzas con el gremio hotelero promocionan a las agencias de viajes planes con precios bajos o innovando con las noches de hotelería dando una noche gratis por cada dos noches, para que el turismo este más al alcance de los colombianos, teniendo en las ganancias netas disminuiría pero podría existir mayor demanda por parte de los colombianos con medianos ingresos, aumentando el PIB por la cantidad de planes turísticos vendidos.

\section{Conclusiones}

En definitiva, esta pandemia ha dejado a casi todas las ramas de actividad de la nación perjudicadas, especialmente al sector turismo, en donde se ha visto una disminución contundente en el PIB de la nación incluso algunos negativos, perjudicando a cada uno de los trabajadores de estos sectores reduciendo el flujo de caja en la nación pues al haber menos ingresos en los hogares colombianos, estos adquirirán en menor cuantía la oferta que el mercado otorgue.

\section{Referencias}

Altman, E.I. (1968), "Financial ratios, discriminant analysis and the prediction of corporatefailure", Journal of Finance, Vol. 23 No. 4, pp. 589-609.

Asociación Nacional de Empresarios en Colombia. Publicaciones Económicas. Consultado el 01 de junio del 2020. Disponible en: http://www. andi.com.co/Home/Pagina/1049-normatividady-guias-de-orientacion-empr

Cámara de Colombiana de Comercio Electrónico. Impacto del COVID-19 sobre el comercio electrónico en Colomba. Consultado el 01 de junio del 2020. Disponible en: https://www. ccce.org.co/wp-content/uploads/2017/06/ Informe-Impacto-

Covid.pdf?utm_source=Icommarketing\&utm medium $=$ email\&utm_content $=$ Informe + Co vid-19\&utm_campaign=Icommarketing + +Boletin+economico+-+Boletin+COVID-19

Comisión económica para América Latina y el Caribe. Informes COVID-19. Consultado el 01 de junio del 2020. Disponible en: https://www.cepal.org/es/publicaciones/ informesCOVID19

FMI (2020): "World Economic The Great Lockdown", Washington (abril). Disponible en: 


\section{4}

https://www.imf.org/en/publications/weo.

Federación de Desarrollo, centro de investigación económica y social. Tendencias económicas. Consultado el 01 de junio del 2020. Disponible en: https://www.fedesarrollo.org.co/ publicacionesInstituto Belisario Domínguez Senado De La República (2020). implicaciones económicas de la pandemia por covid-19 y opciones de política. consultado el 18 de junio del 2020.

Keynes, J. M. (1936) "Teoría General de la ocupación, el interés y el dinero." Traducción Fondo de Cultura Económica. México. 1943

López Laborda Julio. 2020. Consideraciones sobre finanzas públicas y COVID-19: Bastantes interrogantes y algunas certezas. Universidad de Zaragoza y Fedea. Madrid- España. consultado el 02 de junio del 2020.

Ministerio de Comercio, Industria y Turismo, Decreto 557 de 2020. Consultado el 02 de junio del 2020. Disponible e $\mathrm{n}$ : https:/dapre.presidencia.gov.co/normativa/ normativa/DECRETO\%20557\%20DEL\%2015 \%20DE\%20JUNIO\%20DE\%202020.pdf

Ministerio de Comercio, Industria y Turismo, Decreto 462 de 2020. Consultado el 02 de junio del 2020. Disponible e $n$ : https:/dapre.presidencia.gov.co/normativa/ normativa/DECRETO\%20462\%20DEL\%2022 \%20DE\%20MARZO\%20DE\%202020.pdf

Ministerio de Comercio, Industria y Turismo, Decreto 397 de 2020. Consultado el 01 de junio del 2020. Disponible e n : https://apre.presidencia.gov.co/normativa/ normativa/DECRETO\%20397\%20DEL\%2013 \%20DE\%20MARZO\%20DE\%202020.pdf

Surico, P. \& Andrea Galeotti. (2020). The economics of a pandemic: the case of Covid-
19. Wheeler Institute for Business and Development. London Business School.

Pulido Arbelaez L.N. y Cabello Beltrán E.A. (2020). Análisis del impacto económico del coronavirus en el sector turismo en Colombia Ensayo académico para optar por el título de Especialista en Administración Financiera. Tesis de pregrado de la Universidad Católica de Colombia 